\title{
Is there a Problem about Intentionality?*
}

(Erkenntnis 51 (1996), 1-23)

Ansgar Beckermann

1.

The crucial point of the mind-body-problem appears to be that mental phenomena (events, properties, states) seem to have features which at first sight make it impossible to integrate these phenomena into a naturalistic world view, i.e. to identify them with, or to reduce them to, physical phenomena. ${ }^{1}$ In the contemporary discussion, there are mainly two critical features which are important in this context. The first of these is the feature of intentional states, e.g. beliefs and desires, to have a representational or semantic content. The problem of the naturalization of these states I will call the problem of intentionality. The second critical feature is the property of other mental states, e.g. perceptions and sensations, to have a qualitative aspect, i.e. that it is somehow, or feels in a characteristic way, to be in one of those states. The problem of the naturalization of these states is generally called the qualia-problem.

In this paper my intention is to concentrate on the first of the two problems and to argue for the thesis that this problem is an artefact. In my opinion, intentional states do not possess any mysterious feature which could render their naturalization problematic. This only appears to be so. And this appearance ensues from the way in which we ascribe intentional states; i.e. from the vocabulary which we use to ascribe these states to human beings and some higher animals. My claim is that the intentional vocabulary should be interpreted in terms of measurement theory, i.e. analogously to the vocabulary of metric concepts with which we ascribe physical quantities. If one takes this interpretation as a starting point, there no longer exists any reason to assume that intentional states have a special and somehow mysterious feature which, in a sense, forms the basis of our practice of ascribing these states by using intentional predicates. For, to assume this would be comparable to claiming that we ascribe physical quantities by predicates like ' $x$ has a mass of $2 \mathrm{~kg}$ ' because

* This paper is partially based upon my German article (1992d), partially it developes further the considerations put forward in that article. I would like to thank Antonia Barke for translating the paper into English.

1 On the question of how 'reduction' could be understood in this context cf. Beckermann (1992a, 1992b, 1996a, 1996b). 
these properties have the mysterious feature of having a certain numerical content. The measurement theory approach, in my view, has the invaluable advantage to open our eyes to the fact that we do not use intentional vocabulary in ascribing certain mental states because these states have the (mysterious) property of having a semantic content. On the contrary, these states can be said to have a semantic content, if there is such a property at all, only because and insofar as we ascribe them by means of intentional predicates. ${ }^{2}$

I will soon return to this claim. Before I do this, however, I would like to make a few remarks about the character of the problem of intentionality as it is commonly understood.

2.

It appears that this problem was formulated succinctly for the first time by Franz Brentano in the famous passage:

„Every mental phenomenon is characterized by what the Scholastics of the Middle Ages called the intentional (...) inexistence of an object, and what we might call, though not wholly unambiguously, reference to a content, direction toward an object (...), or immanent objectivity. Every mental phenomenon includes something as object within itself, although they do not all do so in the same way. In presentation something is presented, in judgement something is affirmed or denied, in love loved, in hate hated, in desire desired and so on. This intentional in-existence is characteristic exclusively of mental phenomena. No physical phenomenon exhibits anything like it. We can, therefore, define mental phenomena by saying that they are those phenomena which contain an object intentionally within themselves." (Brentano 1924, p. 88f.)

These formulations suggest that a phenomenon is intentional if and only if it is in a certain way directed towards an object, i.e. if it contains in itself something in a non-spatial way as an object. In more recent discussions, however, it has been widely agreed that the intentionality of beliefs, desires, etc. does not consist in a barely intelligible relation to (possibly even non-existing) objects, but rather, that their intentionality consists in their having truth or fulfillment conditions. If one accepts this way of putting the matter, it seems plausible to state Brentano's thesis more precisely thus:

BRENTANO'S THESIS (version 1). Intentional states are characterized by their having a representational content, i.e. truth or fulfillment conditions. Physical states cannot have representational content in this sense. Hence, there exists a principal difference between physical and intentional states.

2 I will have to say a little more on this topic in sec. 8 below. 
In this form, however, Brentano's thesis would be highly implausible. For why should it be the case that physical states cannot have any representational content, hence any truth or fulfillment conditions? After all, we know that linguistic utterances have a representational content. And linguistic utterances are - no matter whether they are realized in patterns of soundwaves or as inscriptions on paper - first of all physical phenomena. In this general form, the thesis that physical phenomena cannot have any truth or fulfillment conditions is certainly not tenable.

This argumentation, however, would miss the point of the problem. For the crucial question in this context is not whether physical phenomena can have a representational content, but rather the question whether the property of having a representational content (truth or fulfillment conditions) is a property which can be explicated in the framework of a naturalistic world view or whether such a property transcends this framework.

Brentano's position as regards this question is obviously characterized by the opinion that having a meaning or a representational content is something which goes beyond the realm of the physical. Therefore his thesis can best be formulated as follows:

BRENTANO'S THESIS (version 2). Intentional states are characterized by their having a representational content, i.e. truth or fulfillment conditions. The property of having a representational content cannot be naturalized, it cannot be explicated in the framework of a purely naturalistic picture of the world. Therefore intentional states are states which go beyond the realm of the physical.

It is clear that in this thesis a challenge lies in wait for every naturalistically minded philosopher - at least if he or she is not tempted to settle the whole problem of intentionality by plunging into eliminativism. For intentionality in the sense of this thesis certainly does not belong to the last and irreducible basic properties of the universe like, for instance, mass and charge. Hence, as a naturalistically minded philosopher, one can only be a realist with respect to intentional states if one is at the same time a reductionist. ${ }^{3}$ The problem which is posed by Brentano's Thesis, i.e. the problem of intentionality as it is conventionally understood, therefore, can be phrased as follows:

The Problem of InTENTIONALITY. Isn't it possible, after all, to naturalize the property of having a representational content? That is to say, can the property of having a representational content $p$ not be identified with, or reduced to, a physical property after all? 
In recent years, many analytical philosophers have tried to solve this problem. In a way this topic almost dominated the discussions in the philosophy of mind. For all that, no solution of the problem has yet been presented which even the most modest majority could agree with. Instead, a great number of - sometimes widely divergent - approaches exist: There is Dretske's approach, which is inspired by information theory, then there is Fodor's theory, in which the idea of causal relations some of which are asymmetrically dependent upon others plays a crucial role and finally, there is the functional-teleological approach as put forward especially by Millikan and Papineau. ${ }^{4}$ If one compares all these attempts to solve the problem of intentionality one gains the following impression: Firstly, in ascribing representational contents ${ }^{5}$ quite a number of factors play an important role, while the individual contributions of each of them often cannot be separated clearly. Secondly, there do not seem to be any clear criteria which would enable us to decide which of the many suggested solutions is right. In other words: Every suggestion is plausible to a certain extent; but with all of them, there rests the impression of a certain arbitrariness.

After this diagnosis at the latest, I think one should suspect that possibly the whole program of the naturalization of intentionality as it is pursued by Dretske, Fodor, Millikan and many others is wrongly conceived. But what exactly is the matter with it? Where does the common error of all these approaches lie?

4 Cf. especially Dretske (1981; 1986), Fodor (1987; 1991), Millikan (1984; 1989) and Papineau $(1985 ;$ 1988). At first sight, the interpretation theoretic account of Haugeland and Cummins seems to be missing in this enumeration. This account however, bears a closer resemblance to the measurement theoretic considerations, with which I will deal in greater detail later, than to the classical attempts to naturalize intentionality which I mention here. (Cf. Cummins 1989, p. 169, ch.9, n.7).

5 Strictly speaking, the expression 'content ascription' is not adequate in the context of Dretske's, Fodor's and Millikan's theories, because these theories presuppose that contents are not ascribed by us, but rather that they are real intrinsic properties of real intentional states - in this instant determined through certain natural conditions. By contrast, the measurement theoretic approach which I will explicate in the following belongs to the group of theories which assume that 'content ascriptions' are not determined by objective facts but are rather based on interpretations. (This point I owe to Peter Lanz). 
In my opinion, the crucial error is to be found in two assumptions. The first assumption is that, when we ascribe certain mental states by means of expressions which normally are formed with the help of 'that'-sentences, we do so because these states have the somehow mysterious property of having a semantic content. And the second, related, assumption is that we must be able to show that this mysterious property has a physical nature if we want to remain naturalists concerning the mental. I think that especially the first of these assumptions rests on a fundamental misunderstanding. A misunderstanding, however, which is very tempting. If one says, for example, $S$ believes that dogs bark, then one seems to say that $S$ is in a state, which, being a belief, firstly belongs to a certain type of intentional states, and secondly, that it differs from other states of the same type, i.e. from other beliefs, by its specific content, namely the content that dogs bark. How then can a state be the belief that dogs bark if it has not the property of having this content?

But this argumentation is too rash: From the fact that in ascribing this state to a system, we use expressions like $\ulcorner$ the belief that $p\urcorner$ it does not at all follow that the use of such an expression can only be correct if the state which is ascribed thus, has, as it were in advance, the special property of having the semantic content $p$. In recent years this point has been emphasized especially by Paul Churchland, who has - in a similar way as some other authors (Field, Stalnaker, Davidson 6 ) - defended the thesis that the vocabulary with which we ascribe intentional states can best be understood analogously to the vocabulary which we use in order to speak about physical quantities, such as length, weight or temperature, and that the role which 'that'-sentences play in the intentional vocabulary, roughly corresponds to the role of numerals in the vocabulary of metric concepts. It is, of course, necessary to be cautious with regard to this analogy, because it might tempt one into blurring the distinctions between these two families of concepts. But at bottom it is right. If one wants to understand the vocabulary which we use to ascribe intentional states, one should, therefore, best begin with an analysis of the vocabulary of metric concepts.

Expressions like ' $x$ has a length of $y \mathrm{~cm}$ ', ' $x$ has a mass of $y \mathrm{~kg}$ ' and ' $x$ has a temperature of $y$ degrees Celsius' may at first sight look as if they would signify relations which exist or fail to exist between spatio-temporal objects and certain numbers. In other words, such expressions seems to have the logical

6 Cf. Churchland (1979, pp. 100-107), Field (1980, p. 114), Stalnaker (1984, pp. 9ff.), Davidson (1974, p. 147; 1989, pp. 9ff.). Cf. also Dennett (1982, pp. 123ff.; 1987b, p. 208) and Matthews (1990). A discussion of the measurement theoretic approach can be found in Lanz (1987, pp. 95-127). Field seems to have been the first one to hold that the measurement theoretic interpretation of intentional predicates could possibly lead to a solution of Brentano's problem (1980, p. 114). 
form $\ulcorner x R y\urcorner$ where $R$ stands for a binary predicate and $x$ and $y$ for expressions which refer to spatio-temporal objects and numbers, respectively. A closer look, however, reveals another possibility - namely, that the mentioned expressions rather have the form $\left\ulcorner F_{y} x\right\urcorner$, where $F$ stands for an operator which applied to an appropriate index expression $y$ - here the expression for a positive real number - results in a monadic predicate $\left\ulcorner F_{y}\right\urcorner .7$ (Such predicates will be called operator-index-predicates in what follows.) If one says 'the cube $a$ has a mass of $2 \mathrm{~kg}$ ', one ascribes a specific property to $a$, a real property which is causally relevant. From the fact that we use an expression for the ascription of this property which contains an expression for the number 2, it does not at all follow, however, that we do so because this property has, as it were in advance, the special and mysterious feature of having the specific numerical content 2, e.g. that it consists in $a$ 's standing in any specific relation to the real number 2.8 If that is so, the question arises, however, why we use at all predicates for the ascription of properties like mass, as well as length or temperature, which contain numerals as an essential part.

In this context one commonly refers to the reflections on the foundation of measurement theory as they were formulated especially by Hempel (1952) and Suppes and Zinnes (1963). These considerations can be succinctly summarized as follows: The concept of mass can be metricized, because in the realm $D$ of objects to which we can apply this concept, there exists a binary empirical relation $H$ (concretely: the relation that holds between two objects $a$ and $b$ if and only if $b$ outweighs or balances $a$ if these two objects are placed into the opposite pans of a beam balance) and an equally binary additive operation $\mathrm{o}$, so that the empirical relational system $\langle D, H, \mathrm{o}\rangle$ corresponds in a specific way to the numerical relational system $\left\langle\mathbf{R}^{+}, \leq,+\right\rangle$of positive real numbers with the 'less than or equal to'-relation and addition. To put it more precisely: The concept of mass can be metricized because there exists a structure-maintaining homomorphism $m$ from the set $D$ into the set $\mathbf{R}^{+}$of positive real numbers, i.e., a mapping for which (1) and (2) hold:

$$
a H b \text { if and only if } m(a) \leq m(b)
$$

7 This account has been explicated in great detail in P. Churchland (1979, pp. 100ff.) To my knowledge this possibility is mentioned for the first time in Quine (1970, sec. 2.11)

8 That $a$ 's property of having a mass of $2 \mathrm{~kg}$ does not consist in $a$ 's standing in a certain relation to the number 2 can easily be seen if we recognize that we can ascribe exactly the same property by means of the expressions ' $x$ has a mass of 2000 g' or ' $x$ has a mass of 70,55 Ounces' although the numerals contained in these expressions designate quite different numbers. 


$$
m(a \circ b)=m(a)+m(b) .^{9}
$$

If we ascribe mass properties by using predicates which contain as index expressions numerals for exactly those numbers which the homomorphism $m$ assigns to the elements of $D$, we can read off from the predicates itself, which position an object occupies in the empirical relational system $\langle D, H, \mathrm{o}\rangle$, and that means especially, in which relations it stands to other objects of the set $D$. If we know of three objects, $a, b$ and $c$ that they have a mass of $2 \mathrm{~kg}, 4 \mathrm{~kg}$, and 6 $\mathrm{kg}$, respectively, we also know that $b$ is heavier than $a$, that $c$ is heavier than $a$ and that $a$ and $b$ together (i.e., $a$ o $b$ ) are as heavy as $c .{ }^{10}$ The main reason for the practice of ascribing e.g. properties of mass by means of operator-indexpredicates, which are generated through the application of operators such as 'has a mass of $y \mathrm{~kg}$ ' to an expression for a positive real number, thus seems to lie in the fact that in these numerical expressions, information is encoded about the position which the object in question occupies in the structure of all objects of set $D$.

4.

The main idea of authors like Churchland, Field, Stalnaker and Davidson is that propositions play a similar role with respect to intentional states as numbers do with respect to physical quantities. Or, to employ the more appropriate formal mode of speech in this context: that the 'that'-sentences by means of which we form the expressions with which we ascribe intentional states, play a role similar to that of the numerical expressions which we use in forming metric concepts. However, after what has been said, this basic idea only seems viable if in the case of intentional states it is also possible to name an empirical and an abstract relational system - in this case obviously a propositional relational system - which can be mapped onto each other in such a way that it is possible to encode information in the 'that'-sentence, chosen to ascribe an intentional

9 The main topics of measurement theory are the so-called representation problem and the uniqueness problem. The first problem concerns the question of the existence of structure-maintaining homomorphisms, i.e. more precisely, the question of which conditions an empirical relational system must fulfill in order for it to be possible to demonstrate that there exists a corresponding numerical relational system into which it can be homomorphically mapped. The uniqueness problem then carries the question further in asking how many such homomorphisms exist under these conditions and in what way they differ from each other.

10 That $a$ is heavier than $b$, is here taken to mean that $b H a$, and not $a H b$ (i.e., that $a$ outweighs $b$ ), and that $a$ is as heavy as $b$, is taken to mean that $a H b$ and $b H a$ (i.e., that $a$ balances $b$ ). 
state, about the position which this state occupies in the corresponding empirical relational system. ${ }^{11}$ The central question in this context, therefore, seems to be: What could the empirical and the abstract relational system look like in the case of intentional states?

At first sight a possible answer to this question seems to suggest itself: The empirical relational system $\langle Z, C\rangle$ consists, in this case, of the set $Z$ of the internal states of a person and of the causal relation $C$ between those states; the abstract relational system $\langle P, I\rangle$, on the other hand, consists of the set $P$ of propositions and the relation $I$ of logical implication which holds between those propositions. This answer seems to suggest itself since on its basis a thesis can be formulated for intentional states which is exactly analogous to the corresponding theses in the realm of physical quantities.

(CT) It is possible and reasonable to ascribe internal states to a system by means of intentional predicates, i.e. by means of predicates which result from the application of an operator such as 'has the belief $y$ ' or 'has the desire $y$ ' to a 'that'-sentence, if there exists an homomorphic mapping $f$ from the set of internal states into the set of propositions, i.e. a mapping for which it holds:

(i) A state $x_{1}$ causes a state $x_{2}$ if and only if the proposition $f\left(x_{1}\right)$ logically implies the proposition $f\left(x_{2}\right)$.

Since this thesis is essentially modeled on the reflections of classical measurement theory, I would like to term it 'Classical Theory' (CT).

Obviously, however, CT suffers from quite a few weaknesses. Firstly, the rationality assumption implicit in it is at the same time too strong and too weak 12 . On the one hand, condition (i) implies that a belief that $p$ causes the belief that $q$ for all $q$ which are logically entailed by $p$. And on the other hand, it also implies that a belief that $p$ can only cause a belief that $q$ if $q$ follows

11 Cf., e.g., the following two passages from Stalnaker (1984):

„What is it about such physical properties as having a certain height or weight that makes it correct to represent them as relations between the thing to which this property is ascribed and a number? The reason we can understand such properties - physical quantities - in this way is that they belong to families of properties which have a structure in common with the real numbers. Because the family of properties which are weights of physical objects has this structure, we can ... use a number to pick a particular one of these properties out of the family.“ (1984, p. 9)

"The analogy suggests that to define a relation between a person or a physical object and a proposition is to define a class of properties with a structure that makes it possible to pick one of the properties out of the class by specifying a proposition." (1984, p. 11)

12 This criticism has already been formulated in a similar way in Lanz (1987, pp. 117ff.). 
logically from $p$. But of course it should be possible that a belief that $p$ causes a belief that $q$ if $p$ just provides good grounds for the belief in $q$, without already implying $q$ logically. And what is more, it should be possible for there to be irrational beliefs, i.e. beliefs which are caused by other beliefs, which do not constitute good reasons for them.

The second weakness of the CT lies in the fact, that it completely ignores the causal relations which exist between the internal states of a system and certain states in its environment or else between the internal states of a system and certain of its actions. If we ascribe certain intentional states to a person $A$, by e.g. saying ' $A$ has the belief that it rains' or ' $A$ has the desire to break the $100 \mathrm{~m}$ sprint record' then we do this, amongst other things, because states of the first kind are in a certain way causally dependent upon it raining, while states of the second type normally cause behavior which is likely to bring $A$ closer to his or her goal. Causal relations between the intentional states of a person and certain states of his or her environment, or certain actions thus also play an important role in the choice of the 'that'-sentences which we use in ascribing intentional states.

There is a third weakness which is closely linked to the one just mentioned. According to CT the content of intentional states is absolutely indetermined, since if there is one mapping $f$, which fulfills condition (i), then there is an infinite number of mappings of this kind. Let us assume for example, that $f$ maps the internal states of a system into the subset $P^{\prime}$ of those propositions whose elements are designated by the 'that'-sentences $\left\ulcorner\right.$ that $\left.p_{1}\right\urcorner$, $\left\ulcorner\right.$ that $\left.p_{2}\right\urcorner$, $\left\ulcorner\right.$ that $\left.p_{3}\right\urcorner$ etc. If everywhere in these expressions we substitute the name 'Konrad Adenauer' by 'Willy Brandt' we get another set of expressions which designate the elements of another subset $P^{\prime \prime}$ of propositions. Obviously, however, the same logical relations obtain between the elements of $P^{\prime \prime}$ as between the corresponding elements of $P^{\prime}$. Hence, if there exists a mapping from the internal states of a system $S$ into the set $P^{\prime}$ that fulfills condition (i), if we uniformly substitute the names and predicates occurring in the 'that'sentences which designate the elements of $P^{\prime}$ by other names and predicates and if we finally take the function $f^{\prime}$ which maps the internal states of $S$ onto the propositions which are designated by the 'that'-sentences which result from this substitution, then $f^{\prime}$ also fulfills condition (i).

The fourth weakness of CT finally consists in the fact that it does not permit a reasonable distinction between different types of intentional states, thus, e.g., it fails to distinguish between beliefs and desires. ${ }^{13}$ This is so

13 For this point as well there is a similar criticism to be found in Lanz (1987, pp. 113ff.). According to Lanz another point in which the analogy between measurement theory and 
because, according to this theory, the function $f$ assigns a proposition $p_{\mathrm{i}}$ to every internal state without telling us whether this state is the belief that $p_{i}$, the desire that $p_{\mathrm{i}}$ or yet another intentional state with the content $p_{\mathrm{i}}$. Here it also becomes clear that CT can claim a certain plausibility only with respect to beliefs, for while it is at least not implausible to assume that someone who believes that $p$ and believes that if $p$, then $q$, also believes that $q$, a similar assumption as regards desires makes little sense.

5 .

I think that the weaknesses just enumerated, demonstrate beyond any doubt, that the first answer to the question, "what could the empirical and abstract relational systems look like in the case of intentional states?' is not a viable one. Does this, however, warrant any more general conclusions pertaining to the analogy between metric and intentional concepts? At first sight, there seem to be only two possible stances. On the one hand, one could argue that from the failure of one answer it certainly does not follow that all other answers are doomed to failure as well. Following this line of thought, one could try to arrive at a satisfying answer to the original question after all through another careful analysis of the situation. On the other hand, one could also come to the conclusion that the difficulties which have arisen with the first answer will in the same - or at least in a similar - way be found in all other possible answers, so that the search for a satisfactory solution must be regarded as a failure at this early stage. If one agrees with the latter diagnosis one might perhaps argue further that from this fact it also follows that the whole idea of an analogy between metric and intentional concepts must be regarded as obsolete.

In my opinion, however, both the outlined stances proceed from a false premise, namely from the assumption that the use of operator-index-predicates is justified if and only if it is possible to name an empirical and an abstract relational system which can be demonstrated to map homomorphically onto one another. This assumption, in turn, seems to rest on the more basic presupposition that we always need a kind of justification for the use of operator-index-predicates, so that if we use such predicates in order to ascribe certain properties or states, there must be a reason in the matter itself which gives us the right to do so.

intentional psychology breaks down is the fact that the empirical relations which must hold amongst objects, in order for certain of their properties to be measurable, can be specified without using numbers, while the corresponding relations between intentional states cannot be specified without recourse to propositions. (Cf. 1987, pp. 107ff.). 
I think, however, that this latter assumption is wrong and that on the contrary we are entirely free to use any predicate we like for ascribing any property. If that is so, however, the question at issue ceases to be 'What is it, concerning the ascribed properties or states, that justifies us in using operatorindex-predicates for their ascription?' but rather the less ambitious one: 'Which motives do we have in proceeding like this in one case and like that in another?' This means that the focus has shifted: The question now is only one of what we gain by preferring to use such predicates rather than others in certain cases.

For the moment I would like to illustrate my point by using a simple example. As everyone knows, we usually ascribe properties of color with expressions like ' $x$ is blue', ' $x$ is green', ' $x$ is red', etc. As far as I can see, however, we could just as well use the following expressions for this purpose: ' $x$ has color 1', ' $x$ has color 2', ' $x$ has color 3'.14 Whether we use operatorindex-predicates for the ascription of properties of color is entirely up to us nothing forces us to do so and nothing prevents us from doing so, either. Normally we do not do it, because it does not bring any advantage; but even that does not mean that we could not do it.

Now, what could the specific advantage of the use of operator-indexpredicates be? This question can lead us to consider afresh the meaning of the fact, that with respect to metric concepts it is always possible to name an empirical and an abstract relational system, which - as can be shown by means of suitable representation theorems - can be homomorphically mapped onto one another.

If the considerations sketched in the last paragraph are correct, we do not need any justification for the use of operator-index-predicates, hence a fortiori, we do not need justification by proving the existence of suitable empirical and numerical relational systems or by demonstrating corresponding representation theorems. However, proofs of this kind do have their merits, but these lie solely in the fact that they inform us that we can tell from the respective index expressions what position the object in question occupies in the structure of all objects of set $D$. As a matter of fact, therefore, proofs of this kind provide us with information about the advantage we can gain, under these conditions, from using certain metric predicates.

This gain, however, is not the only benefit - and in my opinion too little attention is often given to this point - which we can reap from the use of metric predicates in ascribing certain properties. Let us use once more the physical quantity mass to illustrate this. I already mentioned the fact, that if we know of

14 In this case we would - speaking measurement theoretically - measure color at nominal scale level. 
three objects, $a, b$ and $c$ that they have a mass of $2 \mathrm{~kg}, 4 \mathrm{~kg}$, and $6 \mathrm{~kg}$, respectively, we also know - due to the representation theorem which holds for extensive quantities - that $b$ is heavier than $a$, that $c$ is heavier than $a$ and that $a$ and $b$ together are as heavy as $c$. But that's not all! From Newton's Laws it follows that we need a force for $a$ twice the size as that for $b$, if we wish to accelerate the two objects $a$ and $b$ to the same speed in the same time span, or that $a$ will, in the same time, travel three times the distance $c$ will travel if the same force is applied to them. In other words: The benefit which we can obtain from using metric predicates, in my opinion, mainly stems from the fact that the laws which hold for the properties ascribed by use of these predicates, can often be formulated in a thoroughly systematically satisfying manner by way of reference to relations among numbers and simultaneously quantifying over numbers. The fact that physical laws indeed normally have this form becomes apparent, if we rewrite Newton's second law

$$
F=m \cdot a
$$

in operator-index-form:

$$
(x)(i)(j)\left(F_{i} x \& m_{j} x \rightarrow a_{i / j} x\right) .{ }^{15}
$$

6.

In my opinion, the main benefit we gain from the use of intentional concepts also lies in the fact that, with their help, we can formulate laws for the explanation of human behavior in a way which is economic and systematically satisfying. In the remainder of this paper I will elucidate this point using a rather simplified example. In order to do this, I will assume - as already hinted at - that intentional states have the status of theoretical states, which we postulate because they are useful for explaining behavior. Before moving on to the promised example, I should, therefore, like to shortly address the question: Under which conditions have we reason to postulate theoretical states? 16

The principal answer to this question is: We have reason to postulate theoretical states in all those cases in which a system $S$, which has a reasonably complex behavioral repertoire at its disposal, behaves in systematically different ways in situations of the same type. If, for example, in situations of type $\Phi, S$

15 For sake of simplicity of expression I will neglect the fact that forces as well as accelerations are not scalar quantities, but vectors.

16 Concerning the following paragraphs cf. also Beckermann (1986, pp. 319ff.; 1992c, pp. 162ff.). 
sometimes behaves in the way $x$ and sometimes in the way $y$, then the simple explanation suggests itself that $S$ can be in two different dispositional states $A$ and $B$, so that $A$ is responsible for $S$ 's acting in the way $x$ in situations of type $\Phi$ while $B$ is the cause of $S$ 's exhibiting the behavior $y$ in situations of this kind. In this case we, therefore, can - at least partially - explain the behavior of $S$ by means of the following two laws:

(2) In situations of type $\Phi, S$ behaves in the way $x$ if $S$ is in state $A$.

(3) In situations of type $\Phi, S$ behaves in the way $y$ if $S$ is in state $B$.

This is of course only the simplest case. The assumption of theoretical states becomes much more fruitful, however, if (a) one has additional information concerning the conditions under which $S$ will come to be in these states and if (b) one can explain $S$ 's behavior with as small a set of different states as possible, because the differences in the behavior of $S$ can be explained by the interaction of states rather than by special dispositions for each of the different kinds of behavior.

Thus far the general preliminary remark. Now let us take the following case. Let $S$ be one of the systems which behave in situations of the same type, not always in the same, but rather in systematically different ways. Sometimes $S$ goes where there is water and drinks some water; sometimes $S$ goes to a place where there are bananas and eats some bananas. Obviously, these differences in behavior can be easily explained through the assumption that $S$ can be in two different dispositional states $A$ and $B$ which cause the different behavior. That is, in this simple case the behavior of $S$ can be explained on the basis of the two following behavioral laws:

(4) If $S$ is in the state $A, S$ goes to a place where there is water and drinks some water.

(5) If $S$ is in the state $B, S$ goes to a place where there are bananas and eats some bananas.

However, let us assume further that the case is not quite so simple after all, and that $S$ in some cases, in which we have reason to believe that he is in state $A$, does not go to place $x_{1}$ where there is in fact water, but to a place $x_{2}$ where no water is to be found at all. Even in this case, however, it is not to difficult to explain the behavior of $S$ theoretically. We only have to assume that $S$ can be in two further theoretical states $C$ and $D$ and that the behavior of the system is not explained by means of the laws (4) and (5), but, amongst others, with the help of the following laws:

(4a) If $S$ is in states $A$ and $C$ than $S$ goes to place $x_{1}$ (and tries to drink).

(4b) If $S$ is in states $A$ and $D$ than $S$ goes to place $x_{2}$ (and tries to drink). 
Maybe we even discover that $S$ gets into the state $C$ if the situation in which he finds himself, resembles situations, in which there actually is water in $x_{1}$ and something analogous is true for the state $D$, so that apart from the laws (4a) and (4b) we also have the following laws at our disposal:

(6a) If $S$ finds himself in a situation which, in a certain respect, resembles situations in which there is water in $x_{1}, S$ gets into the state $C$.

(6b) If $S$ finds himself in a situation which, in a certain respect, resembles situations in which there is water in $x_{2}, S$ gets into the state $D$.

These really very rudimentary considerations already show that the explanation of $S$ 's behavior, with a growing behavioral complexity, very soon forces us to postulate a large number of different theoretical states, whose causal relations (a) amongst each other, (b) between the states and the different situations in which $S$ can find himself and (c) between the states and the possible actions of $S$ can only be described in an even larger number of behavioral laws. This point becomes even clearer, if we, e.g., assume that in the state $A, S$ does not always go to either $x_{1}$ or to $x_{2}$, but sometimes even to $x_{3}$ or $x_{4}$ or $x_{5} \ldots$ etc., which has to be explained by means of some more theoretical states $E, F, G$ etc. And things become rather impracticable if we further assume that the same holds for the state $B$ and finally that $S$ does not only always go to water or banana-places, but sometimes also to shadow-places, cave-places, rock-places etc.

Not much imagination is needed to realize how quickly the number of the theoretical states which we need for the explanation of $S$ 's behavior exceeds every manageable figure. And if the behavior of $S$ has reached a certain degree of complexity, I think we do indeed need this large number of theoretical states in order to be able to explain $S$ 's behavior. Although we cannot get by with a smaller number of states, however, the laws, in which the causal relations of those states are expressed, can be drastically reduced and simplified. One only has to notice that the theoretical states which we had to postulate in order to explain $S$ 's behavior can easily be grouped into a small number of types. The states $A$ and $B$, e.g., are - similar to the states which explain $S$ 's going to shadow-places, cave-places or rock-places - characterized by the fact that they usually cause $S$ to go to that place in his environment which possesses a certain feature. All of these states, therefore, can be said to belong to one state type (let's call this type 'Des') and differ only in the characteristic feature which the place is required to have. Hence, the idea suggests itself to introduce operatorindex-predicates in which the operator corresponds to the state type in question and the indices to the different characteristic features. In other words, the state $A$ could be ascribed by means of the predicate ' $D e s_{\text {water }}$ ', the state $B$ by the

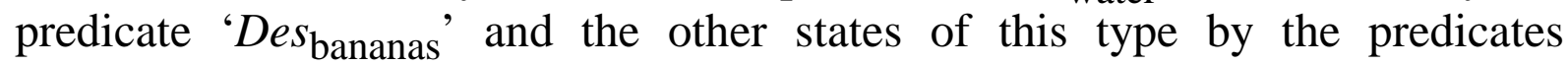
'Des shadow', 'Des $_{\text {cave }}$ ' and 'Des rock '. 
In the next step it is easy to see that the states $C$ and $D$, as well as the states $E, F, G$ etc., also have something in common. These states, therefore, also can be said to belong to a common state type (let us call it ' $B e l$ ') and to differ only in the place where $S$ goes if he is in state $A$ (i.e., in state $D e s_{\text {water }}$ ) and one of these states. Or, to go one step further, if we assume that analogous states $C^{\prime}$, ..., $G^{\prime}$ exist for the case that $S$ is in $B$ (i.e., in state $D e s_{\text {bananas }}$ ), then it is sensible to assume that these states too belong to the state type $\mathrm{Bel}$ and that all states of this type differ not only in one parameter, but in two: the place and the characteristic feature of that place. In this case, it therefore makes good sense to introduce operator-index-predicates with a double-index and to ascribe, e.g., the states $C$ and $D$ with the help of the predicates ' $B e l_{\text {water, } x_{1}}$ ' and ' $B e l_{\text {water, } x_{2}}$ ', the states $C^{\prime}$ and $D^{\prime}$ by means of the predicates ' $B e l_{\text {bananas, } x_{1}}$ ' and ' $B e l_{\text {bananas, } x_{2}}$ ', etc.

The benefit we gain from this new way of ascribing the theoretical states which we need to explain $S$ 's behavior is that we are now able to draw together the laws (4a) and (4b) and all other related laws into one single law, in which we quantify with respect to the appropriate indices:

(4') For all $x$ and $y$ : if $S$ is in the states $D e s_{y}$ and $B e l_{y, x}$, then $S$ goes to place $x$.

The same also applies to the laws (6a), (6b) and all related laws, which can be summed up in the law:

(6') For all $x$ and $y$ : if $S$ gets into a situation, which in certain aspects resembles situations in which $y$ is in place $x$, then $S$ gets into the state $\mathrm{Bel}_{y, x}$.

Hence, the invaluable benefit which even in this simple case results from the introduction of operator-index-predicates is the fact that we can reduce a potentially infinite number of laws to a small manageable number, without having to cut down on the great number of theoretical states which we need in order to explain the behavior of $S$. For the states which are ascribed by means of the predicates ' $B e l_{\text {water, } x_{1}}$ ' and ' $B e l_{\text {water, } x_{2}}$ ', or by means of the predicates ' $B e l_{\text {bananas }, x_{1}}$ ' and ' $B e l_{\text {bananas }, x_{2}}$ ', respectively, are of course still different. Hence, operator-index-predicates facilitate simple formulations without suppressing complexity. 17

17 I would like to acknowledge that the considerations put forward in this section owe a lot to Brian Loar's theory in (1981). Maybe their relation can be characterized as that of being two sides of the same coin. 
The example given in the last paragraph was, of course, chosen so that it at least confers a prima facie plausibility on the assumption that the introduction of intentional predicates has the same advantages as the introduction of the operator-index-predicates $D e s_{y}$ and $B e l_{y, x}$, since it becomes clear in the example which kind of behavior suggests or even demands the introduction of operatorindex-predicates of this kind. And, in my view, the beings whose behavior we explain in an intentional way display exactly this type of behavior - of course in an even much more complex form. Hence, the reason for using intentional predicates is not that the states ascribed by means of these predicates have the mysterious property of having a semantic content. Rather, we use these predicates because it is only with their help that we can formulate relatively simple and manageable behavioral laws. Without this vocabulary we would simply drown in a flood of laws.

As a consequence, however, it follows that there really is no problem of intentionality understood in the traditional way. For the traditional formulation of this problem precisely presupposes that intentional states have a property whose naturalizability is doubtful, viz. the property of having a certain semantic content. The challenge for the naturalistically minded philosopher, therefore, consisted in finding a convincing naturalistic analysis of this property.

I believe, however, that once we see that we do not use intentional predicates because the states ascribed this way possess the mysterious property of having a semantic content, but rather because we need these predicates in order to formulate relatively simple and manageable behavioral laws, we do no longer have the feeling that there is something mysterious about intentional states that stands in need of naturalization. Once we understand the real reason for using intentional predicates, we notice that there is no more reason for the assumption that the states which we ascribe by means of these predicates really differ from 'normal' theoretical states in possessing the mysterious property of having a semantic content than for the assumption that the property we ascribe by means of the predicate ' $x$ has a mass of $2 \mathrm{~kg}$ ' differs from 'normal' properties by its having a mysterious numerical content. Anyone claiming the latter would be guilty of an invalid projection from the linguistic onto the factual level. He would conclude from the fact that we use a predicate for the ascription of this property which contains a numerical expression, that the property ascribed thus has a specific numerical content, that it, e.g., consists in $x$ 's standing in some special relation to the number 2 . This kind of inference, however, is obviously invalid.

In a completely analogous manner, however, those who believe that there is a problem of intentionality, proceed from the fact that in ascribing 
intentional states we use expressions which contain 'that'-sentences to the conclusion that the states ascribed thus possess the mysterious property of having a semantic content. That this inference is invalid, too, is not only shown through the example of metric concepts, but also through the arguments outlined above, since the real reasons why we use intentional predicates have nothing to do with any semantic content which the states ascribed by using those predicates allegedly possess.

I, therefore, conclude that there simply is no problem of intentionality if this is understood as the problem of finding a naturalistic account for some very special feature of some of our mental states, the feature of having a semantic or representational content. This problem is an artefact which results from a wrong analysis of the reasons which are responsible for our using a vocabulary in which 'that'-sentences play an essential role, in order to explain the behavior of e.g. persons. The analysis is wrong, because the reason for using intentional predicates is not that the theoretical states ascribed with their help have some mysterious feature, but solely that only the use of this specific form of operatorindex-predicates allows a simple and surveyable formulation of the corresponding behavioral laws. If there is a problem at all, it is not how (traditionally understood) intentional states come into the world, but at most, how the extremely complex behavior is possible for the explanation of which it is reasonable to use intentional predicates.

8.

Against this conclusion, however, one might raise the following objection. 18 What you have done so far is to argue that from the fact that we measure physical quantities, e.g. the weights of bodies, with the help of numbers it does not follow at all that we thereby assign numerical content to those bodies. Thus, by analogy, we should not assume that we ascribe semantic or representational content by using intentional vocabulary in the ascription of propositional attitudes. But what could it mean for bodies to have numerical content? Maybe not more than that arithmetical vocabulary can be applied to bodies. Similarly, having semantic content may amount to no more than the applicability of intentional vocabulary. Our beliefs may be true or false, our desires fulfilled or unfulfilled. What else could it mean to have semantic content?

This may sound like a powerful objection, and yet, in my view, it is at least half way to unconditional surrender. Let me explain. To think that the problem of intentionality is one of the issues that makes the mind-body problem 
puzzling, is to assume that there is something very special about intentionality, something which poses a real problem to anyone who tries to find a naturalistic analysis of the mind. Philosophers who think that the problem of intentionality is a deep problem, thus, must hold that having a representational or semantic content is a very peculiar feature of some of our mental states, a feature which cannot be naturalized in a simple and easy manner.

My aim was to show that there is no reason to assume that the states we ascribe by using intentional predicates do in fact possess such a feature. But from this it certainly does not follow that intentional predicates do not apply to people. My account, therefore, does not imply that intentional states do not have representational or semantic content if this is supposed to mean no more than that intentional predicates, at least sometimes, do apply to people like you and me.

We have to realize that by now we are confronted with two quite different views about what having a representational or semantic content may consist in. According to the first view having a representational or semantic content is a mysterious and elusive feature of some of our mental states; according to the second view it amounts to no more than the applicability of intentional vocabulary. What I tried to criticize is the first view, the only view that leads to the conclusion that the problem of intentionality poses a serious problem to any naturalistic analysis of the mind. With regard to the second view I do not see any problems at all, since according to this view the problem of intentionality is by no means more mysterious than the 'problem of physical quantities'.

To put it in other words: There is no problem of intentionality in the sense that there is no need of finding a naturalistic account for a mysterious and elusive feature of some of our mental states. For there is no reason to assume that there is such a feature. There is, however, a problem of intentionality if this is understood as the question under which conditions and for what reasons we use intentional vocabulary in explaining the behavior of certain systems, including ourselves and our fellow humans. But there is no reason to think that it is especially hard or even impossible to find a naturalistic answer to this question. If there is no more to the problem of semantic content than to the problem of numerical content, thus, the least we can say is: The problem of intentionality is not a deep philosophical problem.

These remarks are also at least part of the answer to another question: Isn't the price too high one has to pay for the acceptance of the theory I have suggested? Is this theory not only another version of eliminativism? At first sight this could appear to be so, because one of the main theses of this account is that the states which we ascribe by means of intentional predicates themselves do not possess the feature of having a specific content. If this was a 
defining feature of intentional states, then this theory would imply that such states do not exist. However, consider the following: Would we really be prepared to say that no masses, no lengths, no temperatures and no charges exist, simply because the properties we ascribe by means of the corresponding metric predicates do not have the mysterious feature of having a numerical content? To me that seems to go against every intuition. Masses, lengths, temperatures and charges are the properties which we ascribe with the corresponding metric predicates. And the same holds for intentional states: Intentional states are the states which we ascribe with intentional predicates, independent of whatever other characteristics these states may or may not have. If that is so, then the theory I have put forward is not an eliminativistic one, since, according to it, the states of persons which we ascribe with the help of intentional predicates are just as real as other states. 19

\section{9.}

As a form of coda, I would like to add, that the view I've developed in this paper has the additional advantage of casting new light on a subject which is currently being hotly debated - namely, the present discussion concerning individualism and anti-individualism. If one tries to obtain clarity about the different positions in this debate one arrives in my opinion at the following picture.

Burge's main argument 20 for his anti-individualist stance is that the criteria which in our folk psychological practice underlie the ascription of intentional states, lead to the consequence that under certain conditions we then describe the beliefs of two persons even with the help of different contentsentences if these persons do not differ physically. This happens because

19 On this point cf. also the following passage from Davidson:

„We know there is no contradiction between the temperature of the air being 32 fahrenheit or 0 celsius; there is nothing in this 'relativism' to show that the properties being measured are not 'real' [...] in the light of the considerations put forward here, this [i.e., that either of two different interpretations might correctly be put on the same thought (or utterance) of a person] comes to no more than the recognition that more than one set of one person's utterances might be equally successful in capturing the contents of some else's thoughts and speech. Just as numbers can capture all the empirically significant relations among weights or temperatures in infinitely many different ways, so one person's utterance can capture all the significant features of another person's thoughts and speech in different ways. This fact does not challenge the 'reality' of the attitudes or meanings thus variously reported.“ (Davidson 1989, p. 16) 
according to these criteria, it is not only important what happens within that person but also in which environment he or she lives and has been brought up or which language is spoken by the linguistic community he or she belongs to. If, for example, we say, that Elmar 1 has the belief that he has arthritis in his thigh, while we ascribe the corresponding belief to Elmar 2 in saying that he beliefs that he has tharthritis in his thigh (and all that, although Elmar $_{1}$ and Elmar $_{2}$ according to the assumptions differ neither with respect to their physical properties nor with regard to their history, as far as this history can be told in non-intentional terms), then this is only due to the fact that the word 'arthritis' has a different meaning in Elmar, 1 's linguistic community than it has in the linguistic community Elmar $_{2}$ belongs to. 21

Fodor's main argument against Burge's anti-individualism on the other hand is, that although Burge was right concerning our folk psychological practice of ascription, this only serves to show that a scientific psychology needs to employ different criteria of ascription, since the beliefs we ascribe to Elmar 1 and Elmar $_{2}$ in using different content-sentences 'obviously' have the same causal powers; from a scientific point of view, therefore, the beliefs of Elmar 1 and Elmar $_{2}$ would not be different but (type-)identical. ${ }^{22}$

Now, if Fodor's position is correct, that states which have the same causal powers must be regarded as type-identical and further, if his assumption is correct, that the beliefs of Elmar ${ }_{1}$ and Elmar 2 have the same causal powers, then one is forced to admit that the belief states of Elmar ${ }_{1}$ and Elmar 2 are not different, but type-identical. However, does that also mean that there is anything wrong with using different content-sentences in ascribing those beliefs? If my measurement theoretic interpretation of intentional predicates is correct, the answer to that question is: Not at all. For according to this interpretation, it is quite possible to ascribe the same state with the help of different intentional predicates. If we ascribe a certain belief to Elmar ${ }_{1}$ in saying that he believes that he has arthritis in his thigh we, therefore, may well ascribe to Elmar 2 the same belief by saying he believes that he has tharthritis in his thigh - in the same way as we can ascribe the same mass property to a cube $a$ by saying ' $a$ has a mass of $2 \mathrm{~kg}$ ' and ' $a$ has a mass of $2000 \mathrm{~g}$ '. The reason for choosing different 'that'sentences may only be that we are applying different yardsticks. In the first case we use Elmar ${ }_{1}$ 's linguistic community as a yardstick and in the second case

21 Concerning this example cf. Burge (1979, pp. 77f.).

22 For this point cf. esp. Fodor (1987, ch. 2). In (1986) Burge has tried to show that, contrary to what one should expect according to Fodor's argumentation, scientific psychology de facto does use anti-individualist criteria for the ascription of intentional states. 
Elmar $_{2}$ 's linguistic community. Viewed in this light, it seems a great advantage of the measurement theoretic interpretation of intentional predicates that it does justice to both the arguments of Fodor and Burge and thereby yields - in my eyes - a very satisfying solution to the argument over the individualist or antiindividualist individuation of intentional states. 23

\section{References}

Beckermann, A. (1986) Dennetts Stellung zum Funktionalismus. Erkenntnis 24, pp. 309-341.

Beckermann, A. (1992a) Introduction - Reductive and Nonreductive Physicalism. In: Beckermann/Flohr/Kim (1992), pp. 1-21.

Beckermann, A. (1992b) Supervenience, Emergence, and Reduction. In: Beckermann/Flohr/Kim (1992), pp. 94-118.

Beckermann, A. (1992c) Wie real sind intentionale Zustände? Dennett zwischen Fodor und den Churchlands. In: H.J. Sandkühler (Hg.) Wirklichkeit und Wissen. Wirklichkeits-Konzeptionen in Philosophie und Wissenschaften. Frankfurt/M.: Peter Lang. pp. 151-176.

23 A related consideration is found in Loar (1988). There he demonstrates by means of some very useful examples, that on the one hand, there are cases in which we use the same content-sentences for the ascription of beliefs, although the ascribed beliefs, seen psychologically, are quite different and that on the other hand, there are also cases in which we use different content-sentences in order to ascribe beliefs, which are, viewed psychologically, indistinguishable. Loar concludes from this fact that one must differentiate between 'social' and 'psychological' content. In other words: Like Fodor he arrives at the opinion that apart from the 'broad' social content there must also be a 'narrow' psychological one. In my opinion, however, this conclusion is not justified, since it simply presupposes that two (type-)identical intentional states, which we folkpsychologically ascribe by means of different content-sentences, must have something in common which could be expressed in terms of content just because they are intentional states. According to the measurement theoretic account I've put forward however, the states themselves ascribed by means of intentional predicates have no content. Hence, (type-)identical states a fortiori are not characterized through their having the same content in one way or the other. The measurement theoretic interpretation, therefore, permits a solution of the argument concerning the individualist or anti-individualist individuation of intentional states, which can satisfy both parties without bringing 'narrow' contents into play. In my eyes this is another very satisfactory result. 
Beckermann, A. (1992d) Das Problem der Intentionalität - Naturalistische Lösung oder meßtheoretische Auflösung? Ethik und Sozialwissenschaft 3, pp. 433-447, 502-512, 520-522.

Beckermann, A. (1996a) Eigenschafts-Physikalismus. Zeitschrift für philosophische Forschung.

Beckermann, A. (1996b) Property Physicalism, Reduction and Realization. In: M. Carrier/P. Machamer (eds.) Mindscapes. Philosophy, Science, and the Mind. Konstanz: Universitätsverlag/Pittsburgh: Pittsburgh University Press.

Beckermann, A., Flohr, H. and Kim, J. (eds.) (1992) Emergence or Reduction? - Essays on the Prospects of Nonreductive Physicalism. Berlin/New York: Walter de Gruyter.

Burge, T. (1979) Individualism and the Mental. Midwest Studies in Philosophy 4, pp. 73-121.

Burge, T. (1986) Individualism and Psychology. Philosophical Review 95, pp. 3-45.

Brentano, F. (1924) Psychologie vom empirischen Standpunkt. Hrsgg. von O. Kraus. Leipzig: Meiner Verlag. (English translation: Psychology from an Empirical Standpoint. Ed. by L.L. McAlister, translated by A.C. Rancurello, D.B. Terrell and L.L. McAlister. London: Routledge \& Kegan Paul, 1973.)

Churchland, P.M. (1979) Scientific Realism and the Plasticity of Mind. Cambridge: Cambridge University Press.

Cummins, R. (1989) Meaning and Mental Representation. Cambridge, Mass.: MIT Press.

Davidson, D. (1974) Belief and the Basis of Meaning. Synthese 27, pp. 309323. Reprinted in: D. Davidson, Inquiries into Truth and Meaning. Oxford: Clarendon Press 1984, pp. 141-154.

Davidson, D. (1989) What is Present to the Mind? Grazer Philosophische Studien 36, pp. 3-18.

Dennett, D. (1982) Beyond Belief. In: A. Woodfield (ed.), Thought and Object. Oxford: Clarendon Press, pp. 1-95. Reprinted in: Dennett (1987a), pp. 117202.

Dennett, D. (1987a) The Intentional Stance. Cambridge, Mass.: MIT Press.

Dennett, D. (1987b) About Aboutness, in: Dennett (1987a), pp. 203-211.

Dretske, F. (1981) Knowledge and the Flow of Information. Oxford: Blackwell.

Dretske, F. (1986) Misrepresentation. In: R.J. Bogdan, Belief - Form, Content, and Function. Oxford: Clarendon Press, pp. 17-36. 
Field, H. (1980) Postscript zu 'Mental Representation'. In: N. Block (ed.) Readings in the Philosophy of Psychology. Vol. 1. Cambridge, Mass.: MIT Press, pp. 112-114.

Fodor, J. (1987) Psychosemantics. Cambridge, Mass.: MIT Press.

Fodor, J. (1991) A Theory of Content and Other Essays. Cambridge, Mass.: MIT Press.

Haugeland, J. (1981) Semantic Engines. In: J. Haugeland (ed.) Mind Design. Cambridge, Mass.: MIT Press, pp. 1-34.

Haugeland, J. (1985) Artificial Intelligence. Cambridge, Mass.: MIT Press.

Hempel, C.G. (1952) Fundamentals of Concept Formation in Empirical Science. Chicago.

Lanz, P. (1987) Menschliches Handeln zwischen Kausalität und Rationalität. Frankfurt/M.: Athenäum.

Loar, B. (1981) Mind and Meaning. Cambridge: Cambridge University Press.

Loar, B. (1988) Social Content and Psychological Content. In: R.H. Grimm and D.D. Merrill (eds.) Contents of Thought. Tucson: University of Arizona Press, pp. 99-110.

Matthews, R. (1990) The Measure of Mind, Report No. 57/1990 Research Group on MIND AND BRAIN, ZiF (Bielefeld).

Millikan, R. (1984) Language, Thought, and Other Biological Categories. Cambridge, Mass.: MIT Press.

Millikan, R. (1989) Biosemantics. Journal of Philosophy 86, pp. 281-297.

Quine, W.V.O. (1970) Philosophy of Logic. Englewood Cliffs, NJ: PrenticeHall.

Papineau, D. (1985) Representation and Explanation. Philosophy of Science 51, pp. 550-572.

Papineau, D. (1988) Reality and Representation. Oxford: Basil Blackwell.

Stalnaker, R. (1984) Inquiry. Cambridge, Mass.: MIT Press.

Suppes, P. and J. Zinnes (1963) Basic Measurement Theory. In: R.D. Luce et. al. (eds.) Handbook of Mathematical Psychology, Bd. I. New York. 